\title{
A MATHEMATICA PACKAGE FOR $q$-HOLONOMIC SEQUENCES AND POWER SERIES
}

\author{
MANUEL KAUERS * AND CHRISTOPH KOUTSCHAN ${ }^{\dagger}$
}

\begin{abstract}
We describe a Mathematica package for dealing with $q$-holonomic sequences and power series. The package is intended as a $q$-analogue of the Maple package gfun and the Mathematica package GeneratingFunctions. It provides commands for addition, multiplication, and substitution of these objects, for converting between various representations ( $q$-differential equations, $q$-recurrence equations, $q$-shift equations), for computing sequence terms and power series coefficients, and for guessing recurrence equations given initial terms of a sequence.
\end{abstract}

\section{INTRODUCTION}

Computer algebra packages for dealing with recurrence equations are of great relevance as they make a lot of time-consuming, tedious and errorprone hand computations obsolete. This is why, for instance, computer algebra implementations of algorithms for hypergeometric summation [16, $15,1]$ enjoy a large and still increasing community of users with a broad variety of different backgrounds. Even more so than in the ordinary case, computations in $q$-calculus tend to involve large expressions that can be tackled by computer algebra much faster and much more reliably than by hand. Algorithms for ordinary hypergeometric summation have therefore been generalized to summation for $q$-hypergeometric terms $[22,9,14,17,18]$, and implementations of these algorithms are also widely used.

In contrast, no software package was available so far that would deal with univariate $q$-holonomic sequences and power series like the Maple package gfun [20] or its Mathematica cousin GeneratingFunctions [11] do for ordinary univariate holonomic sequences and power series. The class of these objects is closed under a certain number of operations, and there are algorithms for computing a recurrence/differential equation for the result of such an operation given recurrences/differential equations of the operands ("executing closure properties"). These algorithms are at the heart of gfun and GeneratingFunctions.

The packages have become an indispensable tool for proving special function identities via computer algebra. They proved particularly useful in connection with hypergeometric summation algorithms, but are also used frequently in experimental mathematics, e.g., for automatically guessing recurrence equations from sample values of otherwise unknown sequences. Naturally, a $q$-version of gfun/GeneratingFunctions could considerably facilitate the work with $q$-holonomic objects along the same lines.

It follows from general considerations in Ore rings [5] that the closure properties as well as the corresponding algorithms carry over to the $q$-case. Direct proofs were also given recently by Koepf et al. [8]. The purpose of the present paper is to present a Mathematica implementation of these and other algorithms for univariate $q$-holonomic sequences and power series. Besides executing closure properties, our package allows for converting different representations of sequences and power series to each other, for computing sequence values or coefficients of power series, and for automatically guessing $q$-recurrence equations of $q$-holonomic sequences given by some finite sample of values. Our package is available for download from

$$
\text { http://www.risc.uni-linz.ac.at/research/combinat/software/ }
$$

\footnotetext{
* Partially supported by the Austrian Science Foundation (FWF) grants SFB F1305 and P19462-N18.

$\dagger$ Partially supported by the Austrian Science Foundation (FWF) grants SFB F1305.
} 
In Sections 2-4 of this paper, we give descriptions of the commands provided by our package, along with simple examples and the relevant theoretical background. In Section 5, we present a collection of some typical example applications.

To begin with, we load the package into Mathematica.

$\ln [1]:=<$ qGeneratingFunctions.m

qGeneratingFunctions Package by Christoph Koutschan - (c) RISC Linz - V 1.1 (13.11.2007)

\section{2. $q$-Holonomic Sequences And Power Series}

Let $\mathbb{K}=\mathbb{Q}(q)$ with $q$ transcendental. For a formal power series $a(x) \in \mathbb{K} \llbracket x \rrbracket$, the $q$-derivative is defined via

$$
D_{q} \sum_{n=0}^{\infty} a_{n} x^{n}:=\sum_{n=1}^{\infty} a_{n} \frac{q^{n}-1}{q-1} x^{n-1} .
$$

Definition 1. (1) A sequence $\left(a_{n}\right)$ in $\mathbb{K}$ is called $q$-holonomic if there exist $p, p_{0}, \ldots, p_{r} \in$ $\mathbb{K}[x]$, not all zero, such that

$$
p_{0}\left(q^{n}\right) a_{n}+p_{1}\left(q^{n}\right) a_{n+1}+\cdots+p_{r}\left(q^{n}\right) a_{n+r}=p\left(q^{n}\right) \quad(n \in \mathbb{N}) .
$$

(2) A power series $a(x)$ over $\mathbb{K}$ is called $q$-holonomic if there exist $p, p_{0}, \ldots, p_{r} \in \mathbb{K}[x]$, not all zero, such that

$$
p_{0}(x) a(x)+p_{1}(x) D_{q} a(x)+\cdots+p_{r}(x) D_{q}^{r} a(x)=p(x) .
$$

A $q$-holonomic sequence is input by a $q$-recurrence as above, plus possibly some initial values. If no initial values are given, the results returned by the package will be true for any choice of initial values. In order to define a sequence $a_{n}$ uniquely, one has to supply $r$ consecutive initial values and the value $a_{n+r}$ at each singular point $n$, i.e., where $p_{r}\left(q^{n}\right)$ vanishes. Likewise, a $q$-holonomic power series is input by a $q$-differential equation as above, plus possibly some initial values. Again, initial values may be omitted, and in this case generic results will be produced.

It is not essential to Definition 1 that the defining equations may be inhomogeneous, as any inhomogeneous recurrence or differential equation can easily be replaced by an equivalent homogeneous one whenever necessary.

A sequence $\left(a_{n}\right)$ is $q$-holonomic if and only if its generating function $a(x)=\sum_{n=0}^{\infty} a_{n} x^{n}$ is $q$ holonomic. A specification for $a(x)$ can be computed given a specification for $\left(a_{n}\right)$, and vice versa. Our package provides the commands

$$
\begin{aligned}
& \text { QRE2DE }\left[\text { spec, } \text { fun }_{1}\left[\operatorname{var}_{1}\right], \text { fun }_{2}\left[\text { var }_{2}\right]\right] \\
& \text { QDE2RE }\left[\text { spec }, \text { fun }_{1}\left[\operatorname{var}_{1}\right], \text { fun }_{2}\left[\text { var }_{2}\right]\right]
\end{aligned}
$$

for this purpose. The argument fun $\left[\right.$ var $\left._{1}\right]$ declares the function name and the variable in which the specification of the sequence/power series is given. The argument fun 2 [var 2 ] declares the function name and the variable which should be used in the output specification:

$$
\begin{aligned}
& \ln [2]:=\operatorname{QRE} 2 \mathrm{DE}\left[\left\{a[n]==\left(q^{n}+1\right) a[n-1], a[0]==1\right\}, a[n], A[t]\right] \\
& \text { Out[2]= } \quad\left\{-(q-1) q A^{\prime}[t] t^{2}+(-q t-t+1) A[t]-1==0, A[0]==1\right\} \\
& \ln [3]:=\operatorname{QDE} 2 \operatorname{RE}\left[\left\{-(q-1) A^{\prime}[t] t^{2}+(-q t-t+1) A[t]-1==0, A[0]==1\right\}, A[t], a[n]\right] \\
& \text { Out }[3]=\quad\left\{a[n]==-\left(-q^{n}-1\right) a[n-1], a[0]==1\right\}
\end{aligned}
$$

The $q$-derivative can be entered with the usual prime ( $\prime$ ) for derivatives; this symbol also appears in the output. Internally, however, every prime will be replaced by the symbol QDerivative in order to avoid conflicts with Mathematica's symbol Derivative for the ordinary derivative.

Alternatively, a power series may also be input by a $q$-shift equation, i.e., an equation involving expressions of the form $f[q x], f\left[q^{2} x\right]$, etc. Initial coefficients are referred to via $\langle 1\rangle[f[x]],\langle x\rangle[f[x]]$, $\left\langle x^{2}\right\rangle[f[x]]$, etc. (i.e., AngleBracket[1][f[x]], AngleBracket $[x][f[x]]$, AngleBracket $\left[x^{2}\right][f[x]]$, etc.). Conversion between $q$-shift equations and $q$-differential equations is also supported by the package. In fact, $q$-derivatives and $q$-shifts can always be mixed arbitrarily in input. The abbreviations SE 
and DE are therefore synonymous with respect to input; they only differ with respect to output format.

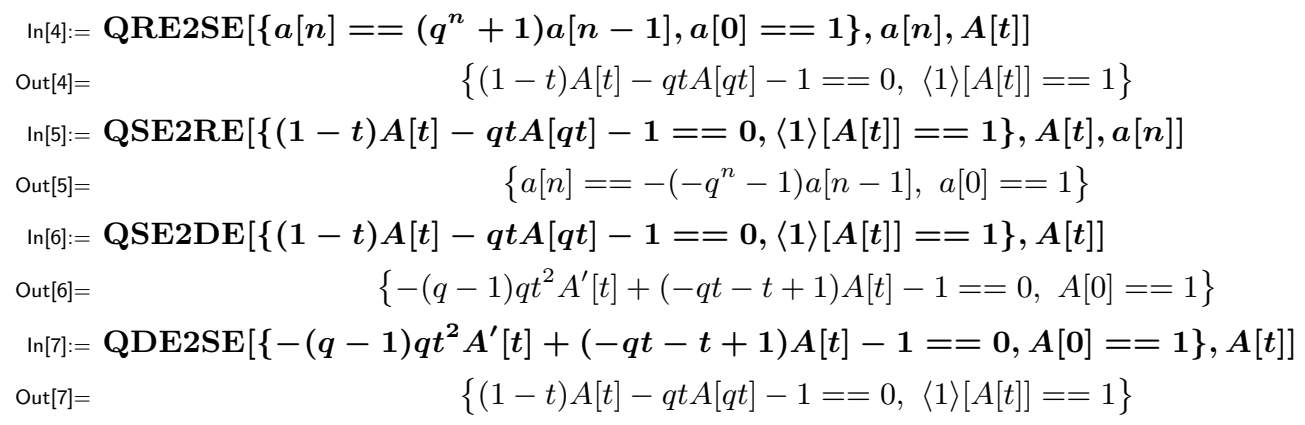

\section{Closure Properties}

We say that the class of $q$-holonomic sequences (or power series) is closed under an operation $\mathcal{F}$ if $\mathcal{F}\left(a_{1}, a_{2}\right)$ is $q$-holonomic whenever $a_{1}, a_{2}$ are. Some useful closure properties are discussed in this section. For all of these, our package provides a command for computing a defining equation for $\mathcal{F}\left(a_{1}, a_{2}\right)$ from defining equations for $a_{1}, a_{2}$. As the proofs are analogous to the ordinary case $[21,11,8]$, we will confine ourselves to provide the proof idea.

\subsection{Sum.}

Theorem 1. (1) If $\left(a_{n}\right)$ and $\left(b_{n}\right)$ are $q$-holonomic sequences, then so is $\left(a_{n}+b_{n}\right)$.

(2) If $a(x)$ and $b(x)$ are $q$-holonomic power series, then so is $a(x)+b(x)$.

Proof idea. (1) If $\left(a_{n}\right)$ and $\left(b_{n}\right)$ are $q$-holonomic, they satisfy homogeneous linear recurrence equations, say of orders $r_{1}$ and $r_{2}$, respectively. Let $c_{n}:=a_{n}+b_{n}$. Using the recurrence equations, every term $a_{n+k}+b_{n+k}(k \in \mathbb{N})$ can be rewritten as a $\mathbb{K}\left(q^{n}\right)$-linear combination of $a_{n+i}$ and $b_{n+j}$ for $i=0, \ldots, r_{1}-1$ and $j=0, \ldots, r_{2}-1$. It follows that at most $r_{1}+r_{2}$ terms $c_{n+k}$ can be linearly independent. Therefore $c_{n}, \ldots, c_{n+r_{1}+r_{2}}$ are linearly dependent. The dependence is the desired recurrence.

(2) Follows from (1).

A specification for the sum of two $q$-holonomic sequences (power series) can be computed from specifications of the two summands, using one of the following commands:

$$
\begin{aligned}
& \text { QREPlus }\left[\text { spec }_{1}, \text { spec }_{2}, \text { fun }[\text { var }]\right], \\
& \text { QDEPlus }\left[\text { spec }_{1}, \text { spec }_{2}, \text { fun }[\text { var }]\right], \\
& \text { QSEPlus }\left[\text { spec }_{1}, \text { spec }_{2}, \text { fun }[\text { var }]\right] .
\end{aligned}
$$

Here, $\operatorname{spec}_{1}$ and $\operatorname{spec}_{2}$ are specifications for the summands, both given using fun as function symbol and var as variable. These symbols will be used again in the output specification of the sum.

\section{Example 1.}

$$
\begin{aligned}
& \ln [8]:=\operatorname{QREPlus}\left[\left\{a[n]==q^{n} a[n-1], a[0]==1\right\},\{a[n]==2 a[n-1], a[0]==1\}, a[n]\right] \\
& \text { Out[8] }=\quad\left\{\left(2 q-q^{n}\right) a[n]==2 q^{n}\left(q^{n}-2\right) a[n-2]-\left(q^{2 n}-4 q\right) a[n-1], a[0]==2, a[1]==q+2\right\} \\
& \operatorname{In}[9]:=\operatorname{QDEPlus}\left[\left\{a^{\prime}[x]-x a[x]==0, a[0]==1\right\},\left\{a^{\prime}[x]-2 a[x]==0, a[0]==1\right\}, a[x]\right] \\
& \text { Out }[9]=\quad\left\{2\left(q x^{2}-2 x+1\right) a[x]+\left(3-q x^{2}\right) a^{\prime}[x]+(x-2) a^{\prime \prime}[x]==0, a[0]==2, a^{\prime}[0]==2\right\} \\
& \ln [10]:=\operatorname{QSEPlus}[\{(1-x) a[q x]-a[x]==0, a[0]==1\},\{a[q x]-a[x]==0, a[0]==1\}, a[x]] \\
& \text { Out[10] }=\quad\left\{-q a[x]+(-x q+q+1) a[q x]+(q x-1) a\left[q^{2} x\right]==0,\langle 1\rangle[a[x]]==2,\langle x\rangle[a[x]]==\frac{1}{q-1}\right\}
\end{aligned}
$$




\subsection{Hadamard Product.}

Theorem 2. (1) If $\left(a_{n}\right)$ and $\left(b_{n}\right)$ are q-holonomic sequences, then so is their Hadamard product $\left(a_{n} b_{n}\right)$.

(2) If $a(x)=\sum_{n=0}^{\infty} a_{n} x^{n}$ and $b(x)=\sum_{n=0}^{\infty} b_{n} x^{n}$ are $q$-holonomic power series, then so is their Hadamard product $a(x) \odot b(x):=\sum_{n=0}^{\infty} a_{n} b_{n} x^{n}$.

Proof idea. (1) If $\left(a_{n}\right)$ and $\left(b_{n}\right)$ are $q$-holonomic, they satisfy homogeneous linear recurrence equations, say of orders $r_{1}$ and $r_{2}$, respectively. Let $c_{n}:=a_{n} b_{n}$. Using the recurrence equations, every term $a_{n+k} b_{n+k}(k \in \mathbb{N})$ can be rewritten as a $\mathbb{K}\left(q^{n}\right)$-linear combination of terms $a_{n+i} b_{n+j}$ for $i=0, \ldots, r_{1}-1$ and $j=0, \ldots, r_{2}-1$. It follows that at most $r_{1} r_{2}$ terms $c_{n+k}$ can be linearly independent. Therefore $c_{n}, \ldots, c_{n+r_{1} r_{2}}$ are linearly dependent. The dependence is the desired recurrence.

(2) Follows from (1).

A specification for the Hadamard product of two $q$-holonomic sequences (power series) can be computed from specifications of the two factors, using one of the following commands:

$$
\begin{aligned}
& \text { QREHadamard }\left[\operatorname{spec}_{1}, \text { spec }_{2}, \text { fun }[\text { var }]\right] \text {, } \\
& \text { QDEHadamard }\left[\operatorname{spec}_{1}, \text { spec }_{2}, \text { fun }[\text { var }]\right] \text {, } \\
& \text { QSEHadamard }\left[\operatorname{spec}_{1}, \text { spec }_{2}, \text { fun }[\text { var }]\right] .
\end{aligned}
$$

\section{Example 2.}

$$
\begin{aligned}
& \ln [11]:=\text { QREHadamard }\left[\left\{a[n]==q^{n} a[n-1], a[0]==1\right\},\{a[n]==2 a[n-1], a[0]==1\}, a[n]\right] \\
& \text { Out[11] }=\quad\left\{a[n]==2 q^{n} a[n-1], a[0]==1\right\} \\
& \operatorname{In}[12]:=\text { QDEHadamard }\left[\left\{a^{\prime}[x]-x a[x]==0, a[0]==1\right\},\left\{a^{\prime}[x]-2 a[x]==0, a[0]==1\right\}, a[x]\right] \\
& \text { Out[12] }=\quad\left\{-x q^{2} a^{(3)}[x]+(-q-1) a^{\prime \prime}[x]+4 a[x]==0, a[0]==1, a^{\prime}[0]==0, a^{\prime \prime}[0]==\frac{4}{q+1}\right\} \\
& \ln [13]:=\text { QSEHadamard }[\{(1-x) a[q x]-a[x]==0, a[0]==1\} \text {, } \\
& \{a[q x]-a[x]==0, a[0]==1\}, a[x]] \\
& \left\{a[x]+(q x-2) a[q x]+(1-q x) a\left[q^{2} x\right]==0,\langle 1\rangle[a[x]]==1,\langle x\rangle[a[x]]==0\right\}
\end{aligned}
$$

\subsection{Cauchy Product.}

Theorem 3. (1) If $\left(a_{n}\right)$ and $\left(b_{n}\right)$ are $q$-holonomic sequences, then so is their Cauchy product $\left(\sum_{k=0}^{n} a_{k} b_{n-k}\right)$.

(2) If $a(x)$ and $b(x)$ are $q$-holonomic power series, then so is their Cauchy product $a(x) b(x)$.

Proof idea. (1) Follows from (2).

(2) If $a(x)$ and $b(x)$ are $q$-holonomic, they satisfy homogeneous linear differential equations, say of orders $r_{1}$ and $r_{2}$, respectively. Let $c(x)=a(x) b(x)$. Using the differential equations, every term $D_{q}^{k} c(x)(k \in \mathbb{N})$ can be rewritten as a $\mathbb{K}(x)$-linear combination of terms $D_{q}^{i} a(x) D_{q}^{j} b(x)$ for $i=0, \ldots, r_{1}-1$ and $j=0, \ldots, r_{2}-1$. It follows that at most $r_{1} r_{2}$ terms $D_{q}^{k} c(x)$ can be linearly independent. Therefore $c_{n}, \ldots, c_{n+r_{1} r_{2}}$ are linearly dependent. The dependence is the desired differential equation.

A specification for the Cauchy product of two $q$-holonomic sequences (power series) can be computed from specifications of the two factors, using one of the following commands:

$$
\begin{aligned}
& \text { QRECauchy }\left[\operatorname{spec}_{1}, \operatorname{spec}_{2}, \text { fun }[\text { var }]\right] \text {, } \\
& \text { QDECauchy }\left[\operatorname{spec}_{1}, \operatorname{spec}_{2}, \text { fun }[\text { var }]\right] \text {, } \\
& \text { QSECauchy }\left[\operatorname{spec}_{1}, \operatorname{spec}_{2}, \text { fun }[\text { var }]\right] \text {. }
\end{aligned}
$$


Example 3.

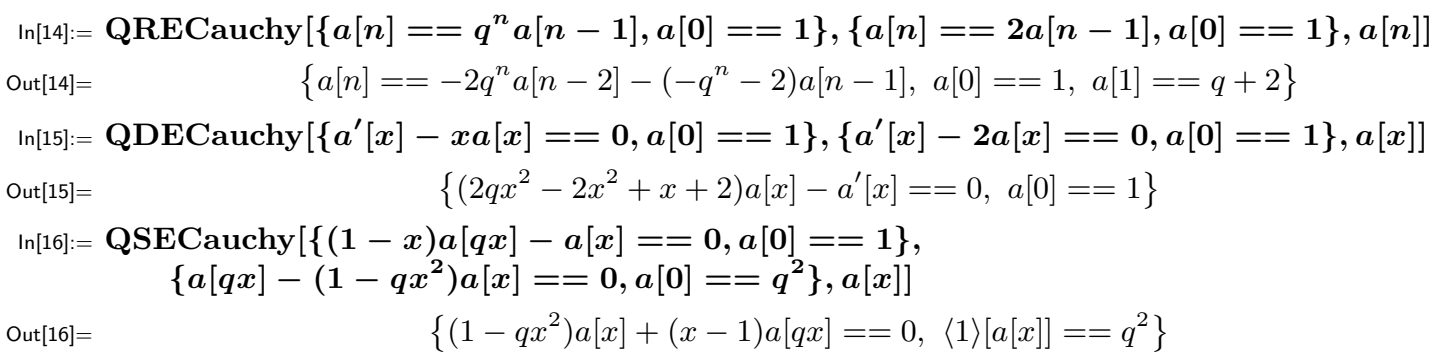

\subsection{Substitution.}

Theorem 4. (1) If $\left(a_{n}\right)$ is a q-holonomic sequence, then so is $\left(a_{\alpha n+\beta}\right)$ for any $\alpha \geq 0, \beta \geq 0$. (2) If $a(x)$ is a q-holonomic power series, then so is $a\left(\lambda x^{\alpha}\right)$ for any $\alpha \in \mathbb{N}$ and any $\lambda \in \mathbb{K}$.

Proof idea. (1) If $\left(a_{n}\right)$ is $q$-holonomic, it satisfies a homogeneous linear recurrence equation, say of order $r$. Let $c_{n}:=a_{\alpha n+\beta}$. Using the recurrence equation, every term $c_{n+k}(k \in \mathbb{N})$ can be rewritten as a $\mathbb{K}\left(q^{n}\right)$-linear combination of $a_{n+\beta+i}$ for $i=0, \ldots, r-1$. It follows that at most $r$ terms $c_{n+k}$ can be linearly independent. Therefore $c_{n}, \ldots, c_{n+r}$ are linearly dependent. The dependence is the desired recurrence.

(2) If $a(x)$ is $q$-holonomic, it satisfies a homogeneous linear $q$-shift equation, say of order $r$. Using this equation, every term $a\left(\lambda q^{\alpha k} x\right)(k \in \mathbb{N})$ can be rewritten as a $\mathbb{K}(x)$-linear combination of $a\left(\lambda q^{i} x\right)$ for $i=0, \ldots, r-1$. It follows that at most $r$ terms $a\left(\lambda q^{\alpha k} x\right)$ can be linearly independent. Therefore, $a(\lambda x), a\left(\lambda q^{\alpha} x\right), \ldots, a\left(\lambda q^{\alpha r} x\right)$ are linearly dependent. After the substitution $x \rightarrow x^{\alpha}$, their dependence yields the desired $q$-shift equation.

The class of ordinary holonomic power series is even closed under substitution with algebraic power series [21]. Statement (2) of the preceding theorem corresponds only to a rather limited special case of this. A $q$-analogue of the more general result is not known.

A specification for the sequence (resp. power series) resulting from substituting $n \mapsto \alpha n+\beta$ (resp. $x \mapsto x^{\alpha}$ ) into a sequence (resp. power series) can be computed from a specification of the latter, by one of the following commands:

$$
\begin{aligned}
& \text { QRESubstitute }[\text { spec, fun }[\text { var }], \alpha \text { var }+\beta] \text {, } \\
& \text { QDESubstitute }\left[\text { spec, fun }[\text { var }], \text { var }^{\alpha}\right] \text {, } \\
& \text { QSESubstitute }\left[\text { spec, fun }[\text { var }], v^{\alpha}{ }^{\alpha}\right] .
\end{aligned}
$$

\section{Example 4.}

$$
\begin{aligned}
& \ln [17]:=\text { QRESubstitute }\left[\left\{a[n]=q^{n} a[n-1]+a[n-2], a[0]==1, a[1]==q\right\}, a[n], 2 n+3\right] \\
& \text { Out }[17]=\quad\left\{a[n]==-q^{2} a[n-2]-\left(-q^{4 n+5}-q^{2}-1\right) a[n-1],\right. \\
& \left.a[0]==q\left(q^{5}+q^{2}+1\right), a[1]==q(q+1)\left(q^{2}+1\right)\left(q^{2}-q+1\right)\left(q^{4}+1\right)\left(q^{5}-q^{3}+1\right)\right\} \\
& \ln [18]:=\text { QDESubstitute }\left[\left\{a^{\prime}[x]-(q-x) a[x]==0, a[0]==1\right\}, a[x], x^{2}\right] \\
& \text { Out }[18]=\left\{x\left(-q^{3} x^{6}+q^{2} x^{6}+q^{4} x^{4}-q^{2} x^{4}-q^{4} x^{2}+q^{3} x^{2}+q^{2} x^{2}+x^{2}-q^{2}-q\right) a[x]+a^{\prime}[x]==0, a[0]==1\right\} \\
& \ln [19]:=\text { QSESubstitute }\left[\{(1-x) a[q x]-a[x]==0, a[0]==1\}, a[x], x^{2}\right] \\
& \text { Out }[19]=\quad\left\{(x-1)(x+1)\left(q x^{2}-1\right) a[q x]-a[x]==0,\langle 1\rangle[a[x]]==1\right\}
\end{aligned}
$$




\section{Additional Functions}

4.1. Evaluation. Given a specification of a $q$-holonomic sequence or power series, we can compute its first terms (resp. coefficients) $a_{0}, \ldots, a_{k}$ using the commands

$$
\begin{aligned}
& \text { QRE2L[ spec, fun }[\text { var }] \text {, range }], \\
& \text { QDE2L[ spec, fun }[\text { var }] \text {, range }], \\
& \text { QSE2L[ spec, fun }[\text { var }] \text {, range }] .
\end{aligned}
$$

\section{Example 5.}

$$
\begin{aligned}
& \ln [20]:=\text { values }=\operatorname{QRE} 2 \mathrm{~L}\left[\left\{a[n+1]==\left(q^{n}+1\right) a[n], a[0]==1\right\}, a[n], 9\right] \\
& \text { Out[20] }=\left\{1, q+1,(q+1)\left(q^{2}+1\right),(q+1)^{2}\left(q^{2}+1\right)\left(q^{2}-q+1\right),(q+1)^{2}\left(q^{2}+1\right)\left(q^{2}-q+1\right)\left(q^{4}+1\right)\right. \text {, } \\
& (q+1)^{3}\left(q^{2}+1\right)\left(q^{2}-q+1\right)\left(q^{4}+1\right)\left(q^{4}-q^{3}+q^{2}-q+1\right), \ldots \\
& (q+1)^{5}\left(q^{2}+1\right)^{2}\left(q^{2}-q+1\right)^{2}\left(q^{4}+1\right)\left(q^{4}-q^{2}+1\right)\left(q^{4}-q^{3}+q^{2}-q+1\right) \\
& \left.\times\left(q^{6}-q^{3}+1\right)\left(q^{6}-q^{5}+q^{4}-q^{3}+q^{2}-q+1\right)\left(q^{8}+1\right)\right\} \\
& \ln [21]:=\operatorname{QDE} 2 \mathrm{~L}\left[\left\{a^{\prime}[x]==(x-q) a[x], a[0]==1\right\}, a[x], 4\right] \\
& \text { Out[21] }=\quad\left\{1,-q, \frac{q^{2}+1}{q+1},-\frac{q\left(q^{2}+q+2\right)}{(q+1)\left(q^{2}+q+1\right)}, \frac{2 q^{4}+2 q^{3}+4 q^{2}+q+1}{(q+1)^{2}\left(q^{2}+1\right)\left(q^{2}+q+1\right)}\right\} \\
& \operatorname{In}[22]:=\operatorname{QSE} 2 \mathrm{~L}[\{a[q x]==(1-x) a[x], a[0]==1\}, a[x], 4] \\
& \text { Out }[2]=\quad\left\{1,-\frac{1}{q-1}, \frac{1}{(q-1)^{2}(q+1)},-\frac{1}{(q-1)^{3}(q+1)\left(q^{2}+q+1\right)}, \frac{1}{(q-1)^{4}(q+1)^{2}\left(q^{2}+1\right)\left(q^{2}+q+1\right)}\right\}
\end{aligned}
$$

4.2. Guessing. A reverse function is provided which makes it possible to guess a recurrence equation satisfied by a list of values:

$$
\text { QREGuess[ list, fun [var ]]. }
$$

We have included this function for the sake of completeness, although there is already more sophisticated guessing machinery for $q$-sequences available [10, 19].

\section{Example 6.}

$\ln [23]:=$ QREGuess[values, $a[n]]$

Out $[23]=$

$$
\left\{-a[n]==-\left(1+q^{n}\right) a[n-1], a[0]==1\right\}
$$

4.3. Equation Manipulation Tools. In general, the recurrence or differential equation returned by our commands are inhomogeneous. At the cost of increasing their order, they can be converted into equivalent homogeneous equations by using one of the commands

$$
\begin{aligned}
& \text { QREHomogeneous [ spec, fun }[\text { var }]], \\
& \text { QDEHomogeneous }[\text { spec, fun }[\text { var }]], \\
& \text { QSEHomogeneous }[\text { spec, fun }[\text { var }]] .
\end{aligned}
$$

\section{Example 7.}

$$
\begin{aligned}
& \ln [24]==\text { QREHomogeneous }\left[\left\{a[n]==\left(1+q^{n}\right) a[n-1]-q^{n}, a[0]==1\right\}, a[n]\right] \\
& \text { Out[24] }=\quad\left\{a[n]==-\left(q^{n}+q\right) a[n-2]-\left(-q^{n}-q-1\right) a[n-1], a[0]==1, a[1]==1\right\} \\
& \ln [25]:=\text { QDEHomogeneous }\left[\left\{a^{\prime}[x]==x a[x]+1, a[0]==1\right\}, a[x]\right] \\
& \text { Out }[25]=\quad\left\{-a[x]-q x a^{\prime}[x]+a^{\prime \prime}[x]==0, a[0]==1, a^{\prime}[0]==1, a^{\prime \prime}[0]==1\right\} \\
& \ln [26]:=\text { QSEHomogeneous }[\{(1-x) a[q x]==x a[x]+1, a[0]==1\}, a[x]] \\
& \text { Out }[26]= \\
& \left\{x a[x]+(-q x+x-1) a[q x]+(1-q x) a\left[q^{2} x\right]==0,\right. \\
& \left.\langle 1\rangle[a[x]]==1,\langle x\rangle[a[x]]==\frac{2}{q},\left\langle x^{2}\right\rangle[a[x]]==\frac{2(q+1)}{q^{3}}\right\}
\end{aligned}
$$




\section{Applications}

5.1. q-Hypergeometric Identities. Recurrence equations satisfied by $q$-hypergeometric sums can be computed with a $q$-analogue of Zeilberger's algorithm implemented in Mathematica by Paule and Riese [14]. This package in connection with ours allows for proving $q$-hypergeometric summation identities effortlessly. As an example, consider Bressoud's identity [4]

$$
\sum_{k=0}^{n} q^{k^{2}}\left[\begin{array}{l}
n \\
k
\end{array}\right]_{q}=\sum_{k=-n}^{n}(-1)^{k} q^{k(5 k+1) / 2}\left[\begin{array}{c}
2 n \\
n+2 k
\end{array}\right]_{q},
$$

where $\left[\begin{array}{l}n \\ k\end{array}\right]_{q}$ denotes the $q$-binomial coefficient. This identity, in connection with the Jacobi triple product identity, implies the Rogers-Ramanujan identities.

We prove this identity by first computing recurrence equations for both sides of the equation.

$\ln [27]:=<<$ qZeil.m

q-Zeilberger Package by Axel Riese - (C) RISC Linz - V $2.42(02 / 18 / 05)$

$\ln [28]:=\operatorname{rec1}=\mathrm{qZeil}\left[q^{k^{2}} \mathrm{qBinomial}[n, k, q],\{k, 0, n\}, n\right]$

Out[28]=

$$
\operatorname{SUM}[n]==-\frac{-q^{2 n}+q^{n+1}-q^{2}-q}{q} \operatorname{SUM}[n-1]-q\left(1-q^{n-1}\right) \operatorname{SUM}[n-2]
$$

$\ln [29]:=\operatorname{rec} 2=\operatorname{qZeil}\left[(-1)^{k} q^{k(5 k+1) / 2} \mathrm{qBinomial}[2 n, n+2 k, q],\{k,-n, n\}, n\right]$

Out[29]=

$$
\begin{aligned}
\operatorname{SUM}[n] & ==\frac{q^{2 n}+q^{2 n+1}+q^{2 n+2}+q^{7}+q^{6}+q^{5}+q^{4}+q^{3}}{q^{3}} \operatorname{SUM}[n-1] \\
& +(\ldots \text { messy } \ldots)+\left(1-q^{2 n-9}\right)\left(1-q^{2 n-8}\right) q^{10} \operatorname{SUM}[n-5]
\end{aligned}
$$

As we obtain different recurrence equations, the identity is not yet evident at this point. Recurrence equations of lower order can often be obtained by applying clever tricks to the sums in question (such as "creative symmetrizing" [13]), but this requires human expertise, which we prefer to avoid. We continue the proof mechanically by combining the above recurrence equations to a recurrence equation satisfied by the difference of left hand side and right hand side.

$\ln [30]:=$ QREPlus $[$ rec1, rec2, SUM $[n]]$

Out $[30]=$

$$
\begin{gathered}
\left\{q^{12} \operatorname{SUM}[n]==\left(q^{2 n}+q^{2 n+1}+q^{2 n+2}+q^{7}+q^{6}+q^{5}+q^{4}+q^{3}\right) q^{9} \operatorname{SUM}[n-1]\right. \\
\left.+(\ldots \text { messy. } . .)-\left(q^{4}-q^{n}\right)\left(q^{n}+q^{4}\right)\left(q^{9}-q^{2 n}\right) q^{5} \operatorname{SUM}[n-5]\right\}
\end{gathered}
$$

As this recurrence has order five, the proof of the identity is completed by checking five initial values, which is easily done. (We may regard it as an irrelevant coincidence that the resulting recurrence is identical to rec2.)

$\ln [31]:=$ Table[

$$
\begin{aligned}
& \operatorname{Sum}\left[q^{k^{2}} \text { qBinomial }[n, k, q],\{k, 0, n\}\right] \\
& \quad==\operatorname{Sum}\left[(-1)^{k} q^{k(5 k+1) / 2} \text { qBinomial }[2 n, n+2 k, q],\{k,-n, n\}\right] \\
& \{n, 0,4\}] / / \text { qSimplify // FullSimplify }
\end{aligned}
$$

Out[31] $=$

\{True, True, True, True, True\}

In the same way, finite versions of further identities like the Goellnitz-Gordon identity or the Rogers-Selberg identity [12] can now be done without any human preprocessing.

5.2. $q$-Trigonometric Functions. Two $q$-analogues of sine and cosine are given by

$$
\begin{array}{ll}
\sin _{q}(x)=\sum_{n=0}^{\infty} \frac{(-1)^{n} x^{2 n+1}}{(q ; q)_{2 n+1}}, & \operatorname{Sin}_{q}(x)=\sum_{n=0}^{\infty} \frac{(-1)^{n} q^{(2 n+1) n} x^{2 n+1}}{(q ; q)_{2 n+1}}, \\
\cos _{q}(x)=\sum_{n=0}^{\infty} \frac{(-1)^{n} x^{2 n}}{(q ; q)_{2 n}}, & \operatorname{Cos}_{q}(x)=\sum_{n=0}^{\infty} \frac{(-1)^{n} q^{(2 n-1) n} x^{2 n}}{(q ; q)_{2 n}} .
\end{array}
$$

Exercise 1.14 of Gasper/Rahman [6] asks for proving the relations

(1) $\sin _{q}(x) \operatorname{Sin}_{q}(x)+\cos _{q}(x) \operatorname{Cos}_{q}(x)=1$,

(2) $\sin _{q}(x) \operatorname{Cos}_{q}(x)-\operatorname{Sin}_{q}(x) \cos _{q}(x)=0$. 
The exercise can be solved by typing the following commands.

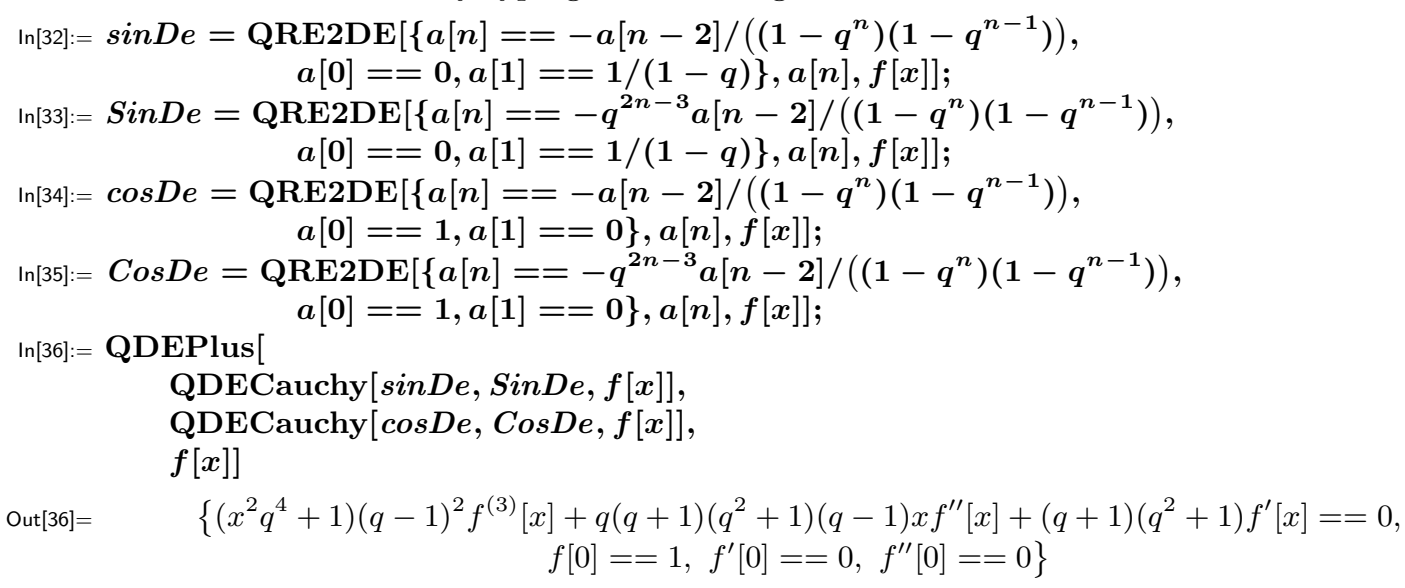

This proves (1), because the differential equation together with $f^{\prime}[0]==f^{\prime \prime}[0]==0$ implies $f^{(n)}[0]==0$ for all $n \geq 1$. Likewise, (2) is proven by

$\ln [37]:=$ QDEPlus[

QDECauchy[sinDe, CosDe, $f[x]]$,

QDECauchy $[\{f[x]==-1\}, \operatorname{SinDe}, \cos D e, f[x]]$,

$f[x]]$

Out[37]=

$$
\begin{gathered}
\left\{\left(x^{2} q^{4}+1\right)(q-1)^{2} f^{(3)}[x]+q(q+1)\left(q^{2}+1\right)(q-1) x f^{\prime \prime}[x]+(q+1)\left(q^{2}+1\right) f^{\prime}[x]==0,\right. \\
\left.f[0]==0, f^{\prime}[0]==0, f^{\prime \prime}[0]==0\right\}
\end{gathered}
$$

5.3. $q$-Fibonacci Numbers. Andrews et al. [3] have given the identity

$$
d_{n} e_{n+1}-d_{n+1} e_{n}=(-1)^{n} q^{\left(\begin{array}{c}
n \\
2
\end{array}\right)} \quad(n \geq 0)
$$

for $\left(d_{n}\right),\left(e_{n}\right)$ defined by

$$
\begin{array}{ll}
d_{n+2}=d_{n+1}+q^{n} d_{n}, & d_{0}=1, d_{1}=0, \\
e_{n+2}=e_{n+1}+q^{n} e_{n}, & e_{0}=0, e_{1}=1 .
\end{array}
$$

For $q=1$, this identity reduces to Cassini's identity for Fibonacci numbers. We can show the identity with our package, by typing the following commands.

$$
\begin{aligned}
& \ln [38]:=\operatorname{defD}=\left\{a[n+2]==a[n+1]+q^{n} a[n], a[0]==1, a[1]==0\right\} ; \\
& \ln [39]:=\operatorname{defE}=\left\{a[n+2]==a[n+1]+q^{n} a[n], a[0]==0, a[1]==1\right\} ; \\
& \ln [40]:=\text { lhs = QREPlus[ } \\
& \text { QREHadamard [defD, QRESubstitute[defE, } a[n], n+1], a[n]] \text {, } \\
& \text { QREHadamard }[\{a[n]==-1\} \text {, QRESubstitute }[\operatorname{defD}, a[n], n+1] \text {, defE, } a[n]] \text {, } \\
& a[n]] \\
& \text { Out }[40]=\quad\left\{q^{11} a[n]==-q^{4 n} a[n-4]+(q+1)\left(q^{n}+q^{2}\right) q^{n+7} a[n-2]+q^{2 n+7} a[n-3]+q^{11} a[n-1],\right. \\
& \left.a[0]==1, a[1]==-1, a[2]==q, a[3]==q^{2}+q-(q+1)\left(q^{2}+1\right)+1\right\} \\
& \ln [41]:=\text { QREPlus }\left[\operatorname{lhs},\left\{a[n]==-q^{n-1} a[n-1], a[0]==-1\right\}, a[n]\right] \\
& \text { Out[41] }=\quad\{a[n]==0\}
\end{aligned}
$$

5.4. Sequences Arising in the Borwein-Conjecture. The first Borwein-conjecture [2] states that if $A_{n}, B_{n}, C_{n}$ are polynomials such that

$$
\prod_{j=1}^{n}\left(1-q^{3 j-2}\right)\left(1-q^{3 j-1}\right)=A_{n}\left(q^{3}\right)-q B_{n}\left(q^{3}\right)-q^{2} C_{n}\left(q^{3}\right),
$$

then these polynomials have positive coefficients. 
The sequences $A_{n}, B_{n}, C_{n}$ are $q$-holonomic, but it is not clear at first glance how to obtain defining recurrence equations for them using closure properties. In such situations, automated guessing is often useful. For example, a recurrence candidate for the $A_{n}$ can be found as follows.

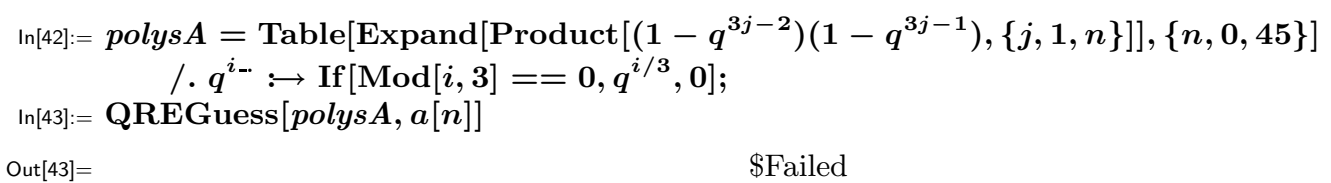

By default, the QREGuess command searches for recurrence equations of order at most two with coefficients quadratically depending on $q^{n}$. The above result asserts that no such recurrence exists for the sequence in question. A higher order and a higher degree for the coefficients has to be tried.

$\ln [44]:=$ QREGuess[polys A, $a[n]$, MaxOrder $\rightarrow$ 4, MaxDegree $\rightarrow 6]$

$$
\begin{gathered}
\left\{-q^{12} a[n]==-\left(q^{2 n}-q^{n+2}+q^{2 n+1}+q^{2 n+2}+q^{5}+q^{4}+q^{3}\right) q^{9} a[n-1]\right. \\
+\left(q^{4 n}-q^{n+6}-q^{n+7}+q^{2 n+4}+2 q^{2 n+5}+q^{2 n+6}-q^{3 n+2}-q^{3 n+3}+q^{4 n+1}+q^{4 n+2}+q^{10}+q^{9}+q^{8}\right) q^{5} a[n-2] \\
-\left(q^{6 n}-q^{n+12}+q^{2 n+10}-q^{3 n+7}-q^{3 n+8}+q^{4 n+5}-q^{5 n+2}+q^{15}\right) a[n-3], \\
\left.a[0]=\left(q^{2}+1\right)\left(q^{2}+q+1\right)\right\}
\end{gathered}
$$

Andrews [2] has given a sum representation for the $A_{n}$ for which the qZeil package delivers the same recurrence, so the recurrence guessed by our package is indeed correct. However, it does not help in proving the conjecture.

5.5. q-Orthogonal Polynomials. The $q$-Hermite polynomials, which are defined by the recurrence equation

$$
2 x H_{n}(x \mid q)=H_{n+1}(x \mid q)+\left(1-q^{n}\right) H_{n-1}(x \mid q), \quad H_{0}(x \mid q)=1, H_{1}(x \mid q)=2 x,
$$

satisfy the identity

$$
\frac{H_{n}(x \mid q)^{2}}{(q ; q)_{n}^{2}}=\sum_{k=0}^{n} \frac{H_{2 n-2 k}(x \mid q)}{(q ; q)_{k}(q ; q)_{n-k}^{2}} .
$$

In order to prove this identity, we first determine a specification for the left hand side.

$$
\begin{aligned}
& \ln [45]:=\operatorname{recH}=\left\{2 x h[n]==h[n+1]+\left(1-q^{n}\right) h[n-1], h[0]==1, h[1]==2 x\right\} ; \\
& \ln [46]:=\operatorname{recFac}=\left\{h[n]==h[n-1] /\left(1-q^{n}\right), h[0]==1\right\} \text {; } \\
& \ln [47]:=\text { recFac2 }=\text { QREHadamard }[\text { recFac, recFac, } h[n]] \text {; } \\
& \ln [48]:=\operatorname{recLHS}=\text { QREHadamard }[\mathrm{recH}, \operatorname{recH}, \operatorname{recFac} 2, h[n]] \\
& \text { Out[48] }=\left\{q\left(q-q^{n}\right)\left(q^{n}-1\right)^{2} h[n]==\left(q-q^{n}\right)\left(q^{n}+4 x^{2} q-q\right) h[n-1]-\left(q^{n}+4 x^{2} q-q\right) q h[n-2]+q^{2} h[n-3]\right. \text {, } \\
& \left.h[0]==1, h[1]==\frac{4 x^{2}}{(q-1)^{2}}, h[2]==\frac{\left(4 x^{2}+q-1\right)^{2}}{(q-1)^{4}(q+1)^{2}}\right\}
\end{aligned}
$$

In a second step we determine a specification for the right hand side.

$\ln [49]:=\operatorname{recH} 2=$ QRESubstitute $[$ recH, $h[n], 2 n]$;

$\ln [50]:=\operatorname{recRHS}=$ QRECauchy[QREHadamard $[\operatorname{recH2}, \operatorname{recFac2}, \boldsymbol{h}[\boldsymbol{n}]], \operatorname{recFac}, \boldsymbol{h}[\boldsymbol{n}]]$

Out [50] $=\left\{-q\left(q-q^{n}\right)\left(q^{n}-1\right)^{2} h[n]==-\left(q-q^{n}\right)\left(q^{n}+4 x^{2} q-q\right) h[n-1]+\left(q^{n}+4 x^{2} q-q\right) q h[n-2]-q^{2} h[n-3]\right.$,

$$
\left.h[0]==1, h[1]==\frac{4 x^{2}}{(q-1)^{2}}, h[2]==\frac{\left(4 x^{2}+q-1\right)^{2}}{(q-1)^{4}(q+1)^{2}}\right\}
$$

As the two specifications agree, the claim follows.

The identity just proven is a special case of the product linearization formula [7, Thm. 3.4]

$$
H_{m}(x \mid q) H_{n}(x \mid q)=\sum_{k=0}^{\min (m, n)} \frac{(q ; q)_{m}(q ; q)_{n}}{(q ; q)_{k}(q ; q)_{m-k}(q ; q)_{n-k}} H_{m+n-2 k}(x \mid q),
$$

which can be also proven automatically in full generality. As this computation is a little more lengthy, we leave it to the reader as an exercise. 


\section{REFERENCES}

[1] Sergei A. Abramov, Jacques J. Carette, Keith O. Geddes, and Ha Q. Le. Telescoping in the context of symbolic summation in Maple. Journal of Symbolic Computation, 38(4):1303-1326, 2004.

[2] George E. Andrews. On a conjecture of Peter Borwein. Journal of Symbolic Computation, 20(5/6):487-501, 1995.

[3] George E. Andrews, Arnold Knopfmacher, and Peter Paule. An infinite family of Engel expansions of RogersRamanujan type. Advances in Applied Mathematics, 25:2-11, 2000.

[4] David M. Bressoud. Some identities for terminating $q$-series. Mathematical Proceedings of the Cabridge Philosophical Society, 89:211-223, 1981.

[5] Frédéric Chyzak and Bruno Salvy. Non-commutative elimination in Ore algebras proves multivariate identities. Journal of Symbolic Computation, 26:187-227, 1998.

[6] George Gasper and Mizan Rahman. Basic Hypergeometric Series. Cambridge University Press, 2nd edition, 2004.

[7] Mourad E. H. Ismail. Lectures on q-orthogonal polynomials. In Special Functions 2000: Current Perspective and Future Directions, pages 179-219, 2000.

[8] Wolfram Koepf, Predrag M. Rajkovic, and Sladjana D. Marinkovic. Functions satisfying $q$-differential equations. Journal of Difference Equations and Applications, 13:621-638, 2007.

[9] Tom H. Koornwinder. On Zeilberger's algorithm and its $q$-analogue. Journal of Computational and Applied Mathematics, 48:91-111, 1993.

[10] Christian Krattenthaler. RATE: A Mathematica guessing machine. Available at http://mat.univie.ac.at/ kratt/rate/rate.html.

[11] Christian Mallinger. Algorithmic manipulations and transformations of univariate holonomic functions and sequences. Master's thesis, J. Kepler University, Linz, August 1996.

[12] Peter Paule. On identities of the Rogers-Ramanujan type. Journal of Mathematical Analysis and Applications, 107:255-284, 1985.

[13] Peter Paule. Short and easy computer proofs of the Rogers-Ramanujan identities and of identities of similar type. Electronic Journal of Combinatorics, 1:1-9, 1994.

[14] Peter Paule and Axel Riese. A Mathematica q-Analogue of Zeilberger's Algorithm Based on an Algebraically Motivated Approach to $q$-Hypergeometric Telescoping. In M.E.H. Ismail and M. Rahman, editors, Special Functions, q-Series and Related Topics, volume 14 of Fields Inst. Commun., pages 179-210. AMS, 1997.

[15] Peter Paule and Markus Schorn. A Mathematica version of Zeilberger's algorithm for proving binomial coefficient identities. Journal of Symbolic Computation, 20(5-6):673-698, 1995.

[16] Marko Petkovšek, Herbert Wilf, and Doron Zeilberger. $A=B$. AK Peters, Ltd., 1997.

[17] Axel Riese. Contributions to Symbolic q-Hypergeometric Summation. PhD thesis, RISC-Linz, 1997.

[18] Axel Riese. qMultiSum - a package for proving $q$-hypergeometric multiple summation identities. Journal of Symbolic Computation, 35:349-376, 2003.

[19] Martin Rubey. Extended Rate, more GFUN. arXiv:math/0702086, 2007.

[20] Bruno Salvy and Paul Zimmermann. Gfun: a Maple package for the manipulation of generating and holonomic functions in one variable. ACM Transactions on Mathematical Software, 20(2):163-177, 1994.

[21] Richard P. Stanley. Enumerative Combinatorics, Volume 2. Cambridge Studies in Advanced Mathematics 62. Cambridge University Press, 1999.

[22] Herb S. Wilf and Doron Zeilberger. An algorithmic proof theory for hypergeometric (ordinary and $q$ ) multisum/integral identities. Inventiones mathematicae, 108:575-633, 1992.

Manuel Kauers, Research Institute for Symbolic Computation, J. Kepler University Linz, Austria

E-mail address: mkauers@risc.uni-linz.ac.at

Christoph Koutschan, Research Institute for Symbolic Computation, J. Kepler University Linz, Austria

E-mail address: ckoutsch@risc.uni-linz.ac.at 